\title{
Lowest Order Squared Rectangles and Squares With the Largest Element Not on the Boundary
}

\author{
By A. J. W. Duijvestijn and P. Leeuw
}

\begin{abstract}
The lowest order squared rectangles and squares with the largest element not on the boundary are presented.
\end{abstract}

Introduction. The problem of dividing a rectangle or a square into a finite number of nonoverlapping squares is frequently mentioned in the literature [1], [2], [3]. A rectangle (or square) that is dissected into $N>1$ nonoverlapping squares is called a squared rectangle (or square) of order $N$. The $N$ squares are the elements of the squared rectangle (or square).

If a squared rectangle (or square) does not contain a smaller squared rectangle, it is called simple. If the elements are all unequal, the squaring is called perfect; otherwise it is called imperfect.

Simple squared rectangles and squares can be derived from 3-connected planar graphs or so-called $c$-nets [1]. The order of a $c$-net is its number of edges. From a $c$-net of order $N+1$, one can obtain squared rectangles or squares of order $N$ [1].

The $c$-nets of order $N+1$ can be constructed out of the $c$-nets of order $N$ using a theorem of Tutte [4]. Given the complete set of 3-connected planar graphs with $N$ edges, the operation of connecting two nonadjacent vertices of each face (of degree 4 or higher) of each one in all possible ways will result in a collection of new graphs with $N+1$ edges which includes every one or its dual, except the wheel which must be added when $N+1$ is even. A wheel of order $B$ is a $c$-net with one vertex of degree $B / 2$ and $B-1$ vertices of degree 3. A computer method for generating $c$-nets is described in [5]. Tutte's generation method gives a set of $c$-nets containing many duplicates (isomorphic graphs). These duplicates are eliminated by the method introduced in [5], and improved in [6], which determines an integer being a characteristic for each graph, invariant under isomorphism.

From a $c$-net one can calculate a squared rectangle or square by placing an electromotive force in one of the branches of the corresponding electrical network containing unit resistances in each of the branches [1]. Squared rectangles or squares are coded using Bouwkamp's code [2].

Two years ago P. J. Federico sent us a simple perfect squared rectangle with the property that the largest element was not at the boundary of the rectangle (see Figure 1). His solution contains 19 elements. Since we have all 3-connected graphs of orders 6 up to and including 22 available on magnetic disk, we could answer the question whether Federico's solution was of lowest possible order.

Received August 18, 1980.

1980 Mathematics Subject Classification. Primary 05B45.

Key words and phrases. Squared rectangles, dissection of rectangles, tiling.

(C) 1981 American Mathematical Society 0025-5718/81/0000-0119/\$02.50 
We searched through the 3-connected graphs of orders 9 to 19, producing all possible squarings of orders up to 18 . We found two simple perfect squared rectangles of order 17 with the largest element not on the boundary. The solutions are given in Figures 2 and 3. The lowest possible order is therefore 17. Of order 18 we found 5 solutions. The Bouwkamp codes of these solutions are given in Table $I$.

From the tables of all (perfect and imperfect) squared squares of orders up to 21 [7], we found one imperfect squared square with the largest element not on the boundary. It is given in Figure 4. The lowest order perfect squared square with the largest element not on the boundary is not known.

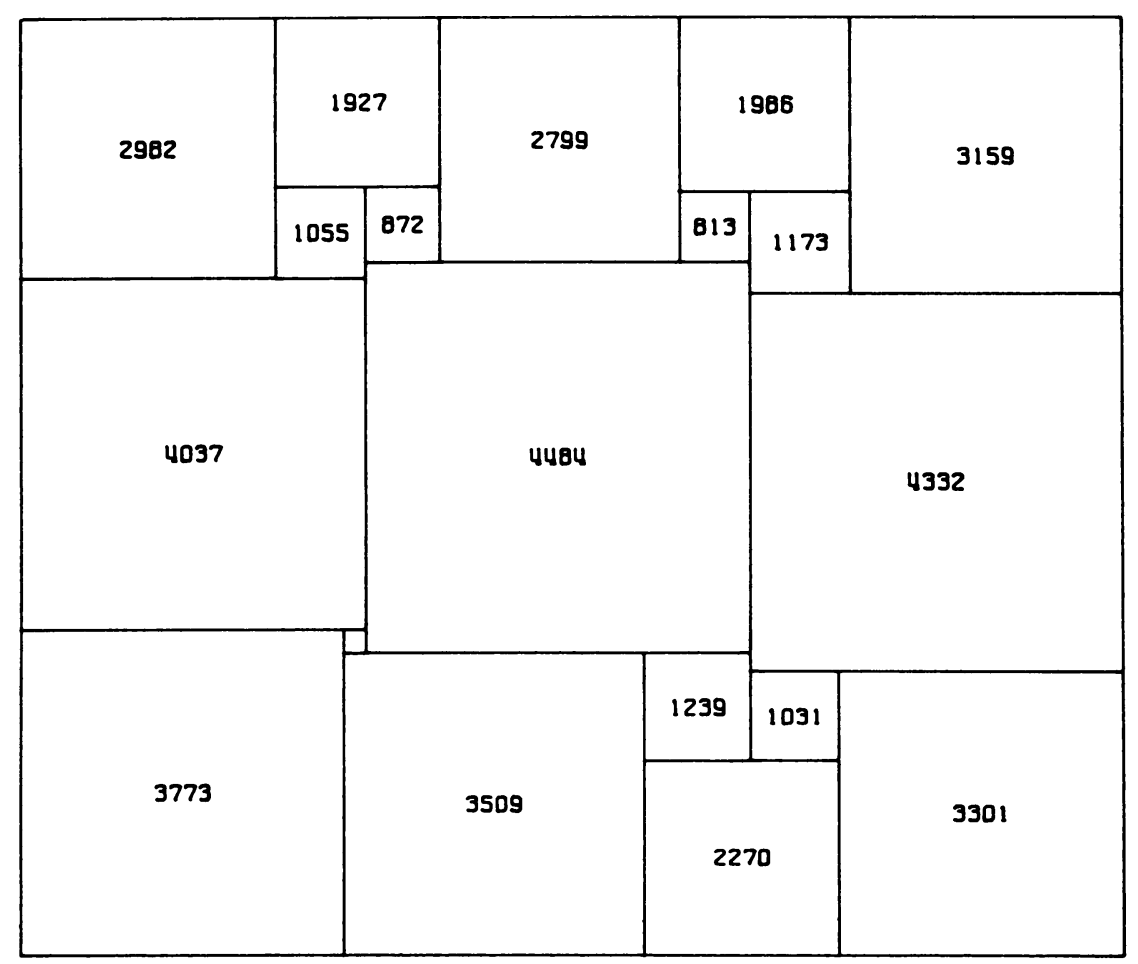

FIGURE 1

Federico's order 19 simple perfect dissection with the largest element not on the boundary 


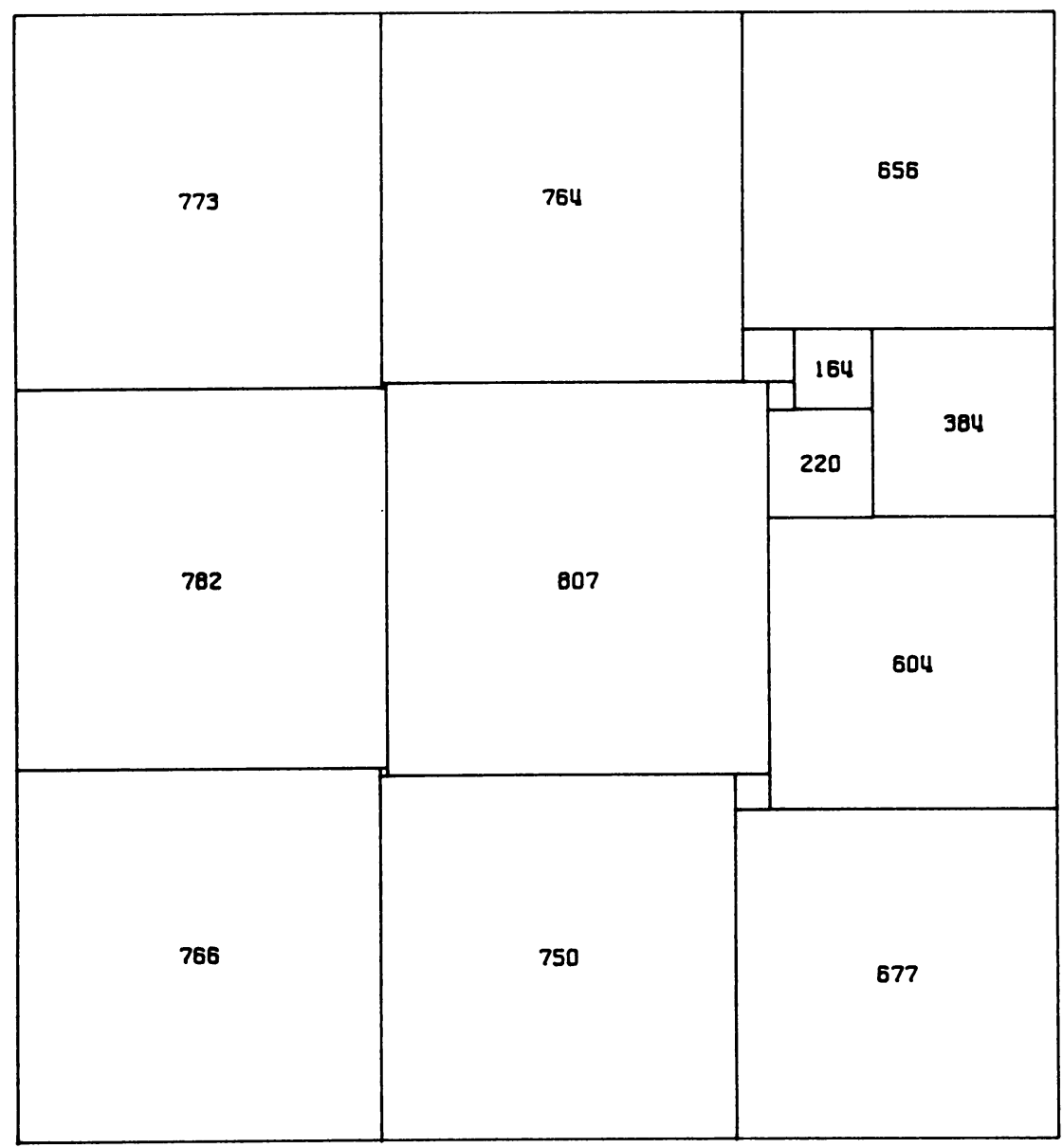

FIGURe 2

First simple perfect dissection of order 17 with the largest element not on the boundary 


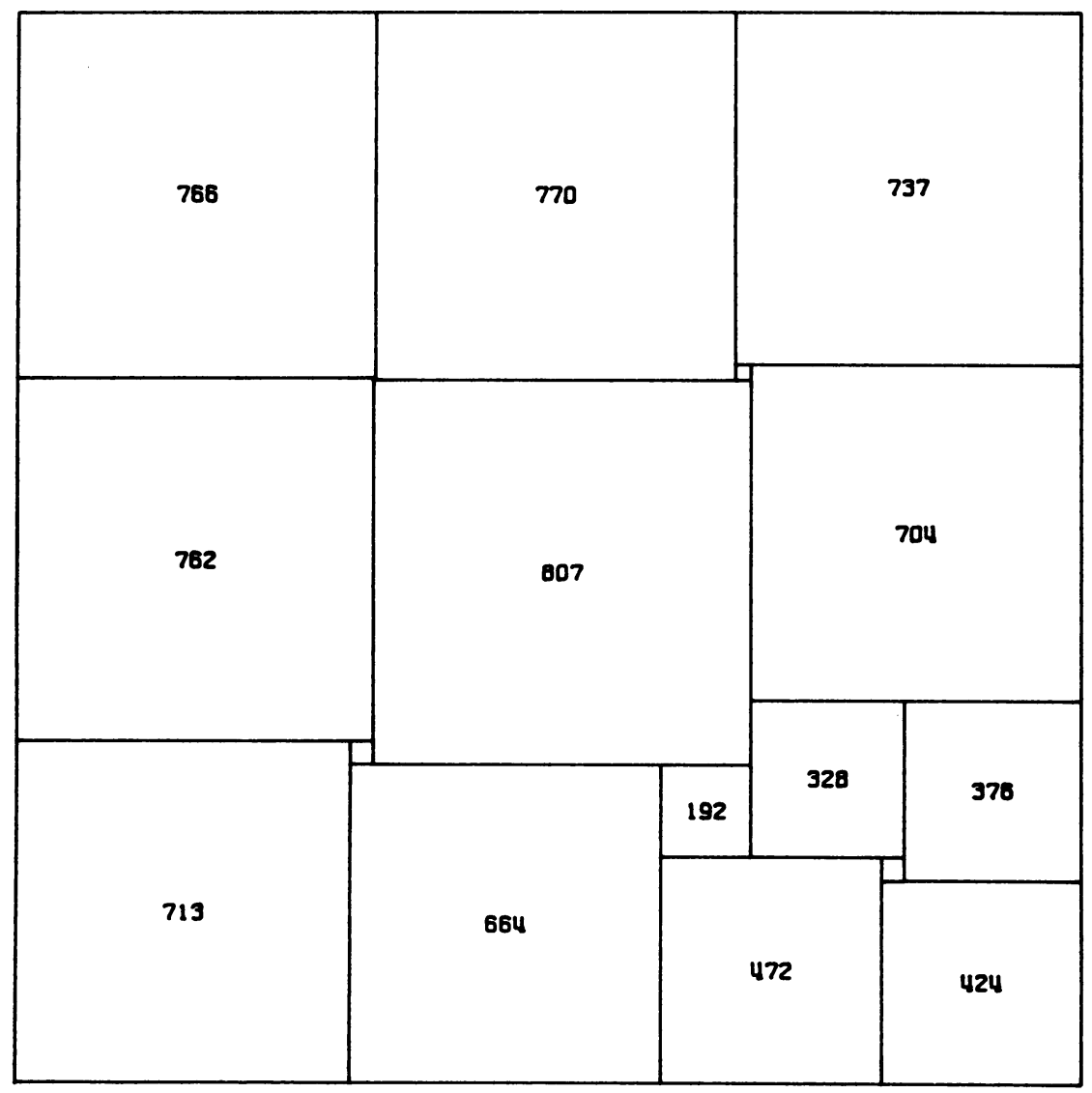

Figure 3

Second simple perfect dissection of order 17 with the largest element not on the boundary 


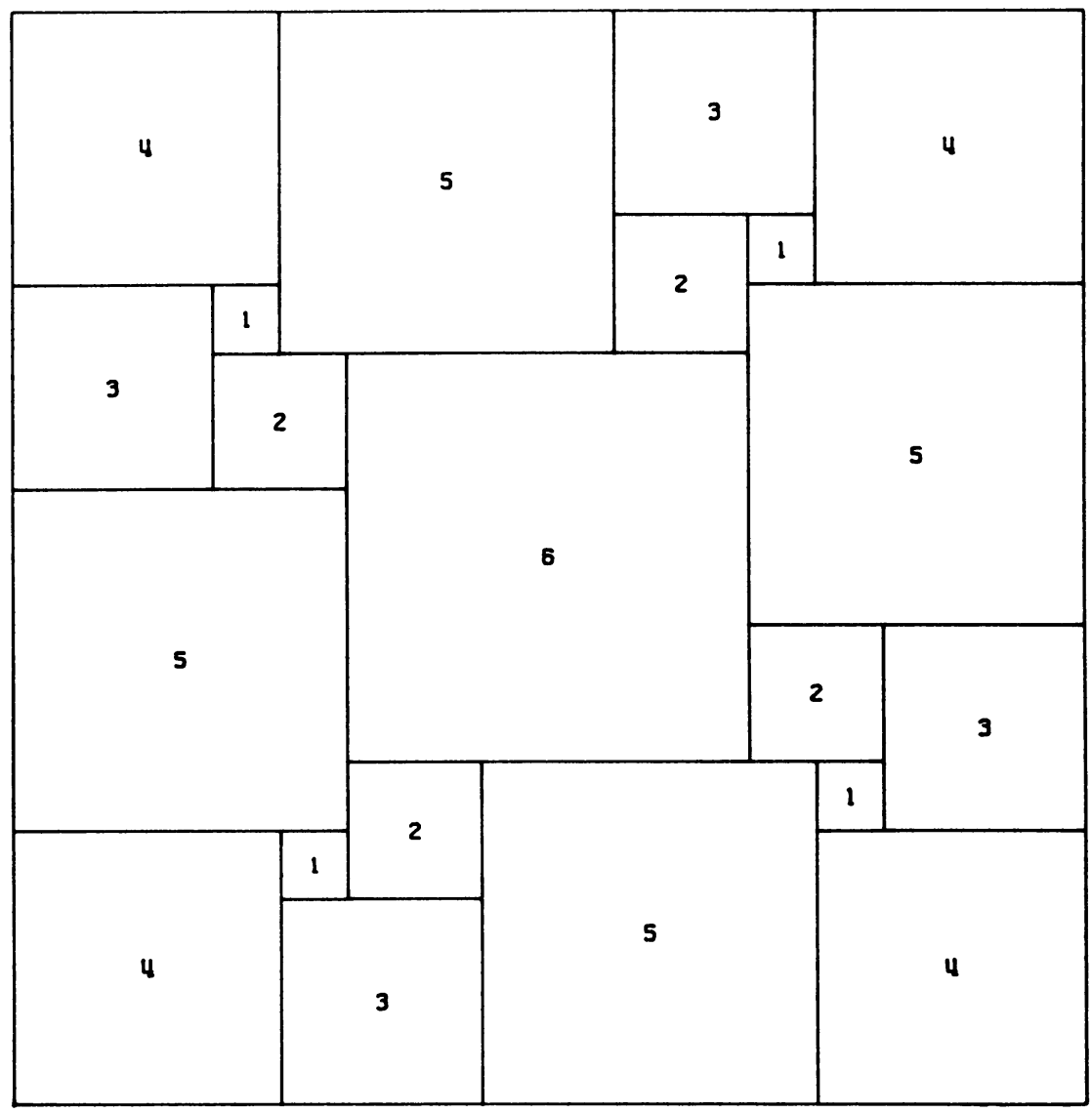

Figure 4

Imperfect squared square of order 21 with the largest element not on the boundary 


\section{TABLE I}

Simple perfect squared rectangles with the largest element not on the boundary

Order 17.

First example: $\quad(773,764,656)(108,164,384)(9,807,56)(782)(220)(604)(766,16)$ $(750,73)(677)$

Second example: $\quad(766,770,737)(33,704)(762,4)(807)(328,376)(713,49)(664,192)$ $(472,48)(424)$

Order 18.

(a): $\quad(842,848,837)(11,826)(836,6)(865)(219,607)(592,209,35)(174,557,169)$ (388)(383)

(b): $\quad(2517,2530,2446)(84,2362)(2504,13)(2627)(349,2013)(1800,704)(568,744,1664)$ $(1096,176)(920)$

(c): $\quad(193,194,190)(4,186)(192,1)(199)(17,169)(160,24,8)(16,56,152)(40)(96)$

(d): $\quad(166,167,163)(4,159)(165,1)(172)(17,65,77)(157,8)(149,48)(101,12)(89)$

(e): $\quad(2446,2442,2253)(189,2064)(4,2627)(2450)(752,1312)(2277,173)(2104,888,560)$ $(328,1544)(1216)$

Department of Applied Mathematics

Twente University of Technology

P. O. Box 217

7500 AE Enschede, The Netherlands

1. R. L. Brooks, C. A. B. SMrth, A. H. Stone \& W. T. Tutre, "The dissection of rectangles into squares," Duke Math. J., v. 7, 1940, pp. 312-340.

2. C. J. BouwkaMP, "On the dissection of rectangles into squares," Proc. Acad. Sci. Amsterdam, v. 49, 1946, pp. 1176-1188; v. 50, 1947, pp. 58-78, $1296-1299$ (= Indag. Math., v. 8, 1946, pp. 724-736; v. 9, 1947, pp. 43-63, 602-625).

3. P. J. FeDerico, "Squaring rectangles and squares," A historical review with annotated bibliography in Graph Theory and Related Topics (Bondy and Murphy, Eds.), Academic Press, New York, 1979, pp. 173-196.

4. W. T. TUTte, "A theory of 3-connected graphs," Proc. Acad. Sci. Amsterdam, v. 64A, 1961 (= Indag. Math., v. 23, 1961, pp. 441-455).

5. A. J. W. DuUvestun, "Electronic computation of squared rectangles," Philips Res. Rep., v. 17, 1962, pp. 523-613.

6. A. J. W. DuUJvestuN, Algorithmic Identification of Graphs and Determination of the Order of the Automorphism Group of a Graph, Memorandum 220, Twente University of Technology, Enschede, The Netherlands, 1978.

7. A. J. W. DuIJvestiJ, Tables of Simple Squared Squares of Orders 13 Through 21 and $2 \times 1$ Rectangles of Orders 17 Through 21, Twente University of Technology, Enschede, The Netherlands, 1979. 